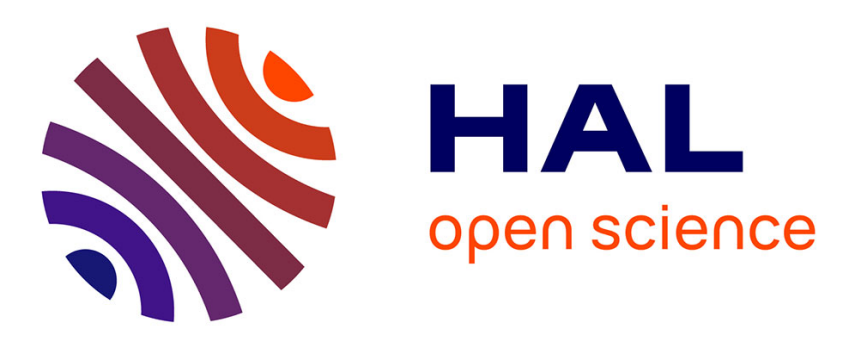

\title{
Single vehicle network versus dispatcher: user assignment in an agent-based model
}

Alexis Poulhès, Jaâfar Berrada

\section{To cite this version:}

Alexis Poulhès, Jaâfar Berrada. Single vehicle network versus dispatcher: user assignment in an agent-based model. Transportmetrica A: Transport Science, 2019, pp.1-23. 10.1080/23249935.2019.1570383 . hal-02042955

\section{HAL Id: hal-02042955 https://hal.science/hal-02042955}

Submitted on 30 Jun 2020

HAL is a multi-disciplinary open access archive for the deposit and dissemination of scientific research documents, whether they are published or not. The documents may come from teaching and research institutions in France or abroad, or from public or private research centers.
L'archive ouverte pluridisciplinaire HAL, est destinée au dépôt et à la diffusion de documents scientifiques de niveau recherche, publiés ou non, émanant des établissements d'enseignement et de recherche français ou étrangers, des laboratoires publics ou privés. 
Single Vehicle Network Versus Dispatcher: User Assignment in an Agent-Based Model

Poulhès Alexis

LVMT, Université Paris-Est, Champs-sur-Marne, France

Alexis.poulhes@enpc.fr

Berrada Jaâfar

VEDECOM, Versailles, France 


\section{Single Vehicle Network Versus Dispatcher: User Assignment in an Agent-Based Model}

The spread of smartphone applications, the negative social impacts of the private car or the potentially revolutionary effect of future autonomous vehicles, are contributing to the emergence of other hybrid mobility systems. Notably, companies like Uber or Lyft have successfully applied the classical dial-a-ride method, in which users are assigned to vehicles in such a way as to minimise the cost function under a set of constraints. In this paper, we construct an agentbased model based on vehicles where demand is assigned through aggregation by origin-destination pair. Two operating strategies are described: in the first, the pick-up strategy is applied at vehicle level, in the second it is applied at global service level, with vehicles being shared dynamically and making extra stops to collect travellers with different destinations. At each iteration time, the set of possible options depending on itinerary and demand, are tested first for already assigned vehicles then for free vehicles. The option associated with the maximum utility is selected to set the vehicle or service strategy. A case study illustrates a comparison between both strategies and evaluates the efficiency of several service choices. A service where vehicles are managed by a dispatcher is more efficient for both users and operator.

Keywords: dispatching, ridesharing, agent-based models, user assignment, dial-aride, dynamic pickup

\section{Introduction}

\subsection{Background}

Demand responsive transportation systems are characterised by their flexibility on spatial and temporal levels. The oldest and most popular form is the taxi, where customer service is based on a simple first-come, first-served rule-based algorithm because of past technological constraints. This strategy, however, is highly inefficient in an overloaded system: when all taxis are busy, whenever a taxi becomes idle, it is immediately dispatched to the longest waiting open request. This approach is based on the pickup and delivery problem. Berbeglia et al. (2010) present a good review of main pickup and delivery problems. In particular, Agatz et al. (2012) present a review for algorithms of dial-a-ride problems and Cordeau et al. (2007) analyze their 
computational performance. Furuhata et al. (2013) identify ridesharing development barriers and then recommend future research directions. Finally, these works suggest improvement in the optimization problem with new constraints or faster optimization or heuristic solutions (Hosni, et al., 2014).

Because of the development of ratio taxi dispatching systems, longest taxi waiting time was included in the dispatching algorithm (Alshamsi, et al., 2009). Today, with the emergence of new mobility supply schemes like Uber, carsharing and ridesharing, taxi dispatching decisions are more often based on real-time geolocalisation systems, so that the vehicle closest to the customer is sent (Lee, et al., 2013; Maciejewski, 2016; Seow, et al., 2010). Maciejewski and Nagel (2013) presented three different strategies for the taxi-dispatching problem with immediate requests. The first adopts a FCFS algorithm to assign the nearest idle taxis to users; the second and third strategies consider idle and en-route drop-off taxis. The third strategy re-assigns demand requests in the taxi queues to other taxis as new information enters the system. Maciejewski et al. (2014) explore collaboration schemes in taxi dispatching between customers, taxi drivers and the dispatcher. They demonstrate that cooperation between the dispatcher and taxi drivers is indispensable, while communication between customers and the dispatcher may be replaced by more sophisticated strategies. Maciejewski et al. (2016) use the assignment framework to dispatch taxis to immediate requests in a large-scale network,, but in a system without ridesharing. Lee et al. (2013) and Salanova et al. (2015) compared the strategies of both booking (i.e. call a dispatcher) and non-booking services (i.e. hailing, taxi ranks). Hörl et al. (2017) assessed the performance of four different dispatching and rebalancing algorithms for the control of an automated mobility-on-demand system. The dispatcher determines an 
optimal bipartite matching between all open requests and available vehicles using the shortest Euclidian distance (Hörl, et al., 2017; Agarwal, 2004).

With the advent of automation technologies, there is now the prospect of services based on autonomous cars (AV). However, the fleet dispatchers in most existing research on autonomous vehicle services use simplistic rules to assign vehicles to passengers. Burns et al. (2013) and Zhang et al. (2015a; 2015b) assigned travellers to the nearest idle and en-route drop-off AV on a FCFS basis. Fagnant and Kockelman (2014), Boesch et al. (2016), Chen et al. (2016) and Zhu et al. (2017) propose similar simplistic rule-based strategies while segmenting the service region into sub-regions and assigning available AVs in the closest sub-region.

AVs will open the doors to new kinds of dispatching strategies, with communication between vehicles about their intentions in the next few seconds resulting in an optimal cooperative system. Another promising form of AV dispatching assumes that vehicles will behave like a taxi driver, i.e. will look for passengers with the sole aim of maximising profit. Few studies have computed the performances of these two forms of dispatching. Lioris (2010) undertakes a discrete-event simulation for ridesharing services with both centralised and decentralised management systems. Nourinejad et al. (2014) suggest an agent-based model for for-hire services applying both centralised and decentralised optimisation algorithms. The results indicate greater savings on user costs and on vehicle kilometres travelled (VKT) when multi-passenger rides are allowed. Hyland et al. (2018) assess the operational performances of six assignment strategies for SAVs with no shared trips (i.e. two simplistic strategies, based on FCFS algorithms, and four optimization-based strategies). The results show that two optimisation-based assignment strategies - (1) strategies that allow en-route pickup 
AVs to be diverted to new traveller requests and (2) strategies that consider en-route drop-off AVs - produce the lowest vehicle travel distances and traveller waiting times.

\subsection{Objective}

In general, vehicle itineraries are calculated in such a way that each new user is considered automatically and independently while the dispatcher looks for a match between the user and all available service vehicles. The time required to calculate matches is therefore restrictive for big operational services. However, unlike classical transit systems, service users do not need to transfer between vehicles and are transported directly to their destination station. The objective of this paper is to reformulate the problem with more flexibility but also to reach an aggregated solution to optimise calculation time.

This paper explores the optimisation of system performance through coordination between vehicles for a shared on-demand mobility service. In particular, we focus on two assignment strategies: the single-vehicle strategy, where the taxi is only concerned with its own performance, and seeks to maximise its utility without consideration for other vehicles in the system; and the dispatching strategy, where the vehicles communicate through a dispatcher and collaborate to maximise the system's total utility. This paper is a development of the model developed by Poulhès et al. (2018), which considers only the first configuration under simplified terms. In addition, it assumes that taxis choose the most pertinent system users from a queue of waiting users. Finally, the objective is that the model should serve as a decision tool that helps to optimise supply resources (i.e. fleet size and vehicle capacity) and user satisfaction (i.e. waiting time). The original contributions of this paper include:

- The description of two assignment strategies and their mathematical formulation. 
- The presentation of the solution of the utility maximisation problem using non-heuristic methods, which encompass all possible combinations of vehicle cooperation.

- Quantitative comparison between the performances of the single vehicle assignment strategy and dispatching.

- Assessment of the role of dispatching from the perspective of passengers and the service provider.

- The application of the model to a real network.

\subsection{Approach}

As previously mentioned, the proposed taxi assignment model is a development of Poulhès et al. (2018). It is based on a consideration of the physical interactions between vehicles, users and the service by cross-simulating vehicle itineraries and the boarding and alighting of booked users at the corresponding stations. This approach differs from classical operational research approaches, where all the physical aspects are combined in a cost function under a set of constraints.

In particular, the model adopts different algorithms depending on the status of the taxis. (1) In the case of assigned vehicles, the itinerary is already defined, so potential new users must fit in with it. Under the single-vehicle strategy, the model determines which users should prioritise the vehicle. Conversely, under the dispatching strategy, several vehicles can potentially consider the same users. The best option for the overall system (users + operators) is determined as the combination of vehicles strategies. (2) Free vehicles serve residual demand by following the same assignment rules.

As can be observed, the vehicle is considered in our model as the decision-make on movement strategies and passenger service priorities. Assignment is therefore carried 
out in accordance with the utility functions of vehicles, which consist of minimum travel time, minimum user waiting time and maximum vehicle occupancy. The model therefore proposes a new formulation of the problem of maximising vehicle utility. In addition, the assignment of passengers to vehicles is controlled directly by vehicle drivers at an aggregated level by origin-destination pair. Finally, the distinction between assigned and free vehicles and the restriction placed on the range of options reduce the complexity of the system.

The model thus examines two coordination configurations by detailing the strategies that vehicles are likely to adopt. Their utility functions are defined and their complexity is calculated.

\subsection{Article structure}

The structure of the paper is as follows. First, we present the framework of the model by defining the supply-side and demand-side entities (Section 2). We provide an overview of the specifications and general scheme of the model (Section 3). This part also explains the associated service framework. Then, we investigate the single vehicle service (Section 4) and cooperative service (Section 5) configurations by defining strategies and utility functions for assigned and free vehicles. Finally, a case study illustrates the theoretical model by testing different supply scenarios (Section 6 and Section 7). The difference between the two approaches is finally evaluated in term of running time and socioeconomic indicators. 


\section{Model framework}

\subsection{Supply side}

\subsubsection{The network}

The network is represented by an oriented graph $\mathrm{G}=(\mathrm{V}, \mathrm{A})$, where $V$ is the node set and $A$ is the link set. The node set $V$ consists of stations $i \in N$ and link intersections $\bar{\imath} \in \bar{N}: V=N \cup \bar{N}$. Boarding and alighting are allowed at stations $i$ but not at link intersections. The link set A contains travel links on which vehicles run. Each travel link $a \in A$ is characterised by a distance value $l_{a}$ and a maximum authorised speed $\overline{v_{a}}$. The other modes (private cars, transit modes, walking...) are not considered within the model.

\subsubsection{The service}

A service consists of a fleet of vehicles $k \in K$ and a set of stations $N$. We consider that the service is station-based - rather than operating through smartphone geo-tracking-in order to structure demand, and to make the service available to users without smartphones as well as more secure and visible.

(i) The stations

The stations are the points where users access and leave the service. Vehicles reach the stations in a single trip. We therefore exclude transfers between two vehicles in a given station, even if this would optimise system performance. In particular, users specify their destination when booking the taxi, which means that their destination is fixed before they board. In addition, users cannot change their destination during the trip. The destination is necessarily the alighting station. Finally, we assume that platforms have infinite length and that vehicle queues are avoided, and also that stations can accommodate an unlimited number of users.

\section{(ii) The vehicles}


For a given vehicle $k$, the current speed on a link $a$ is $v_{a}=\min \left(\overline{v_{a}}, v_{k}\right)$, where $v_{k}$ is the default technical speed of vehicle $k$ and $\overline{v_{a}}$ the authorised speed value (or the exogenous congested speed value on the $\operatorname{arc} a$ ).

The capacity of vehicle $k$ is $\kappa$. At instant $t$, the number of occupied seats is $\overline{\kappa_{b}}(t)$ while the number of available seats (relative capacity) is $\kappa_{b}(t)$. Thus we have $\kappa=\kappa_{b}(t)+\overline{\kappa_{b}}(t)$. We assume that all passengers in the vehicle are seated. We consider two vehicle states:

- Vehicles with a defined itinerary where users are on board or reserved, noted $K_{l}, 0 \leq \kappa_{b}(t) \leq \kappa$. This state is called "assigned vehicle"

- Free vehicle without pre-defined itinerary, noted $K_{e} \kappa_{b}(t)=\kappa$ and called "free vehicle". In this state, vehicles are stationary and waiting for new demand to be assigned. Anticipation of reserved users with repositioning is not considered in this model.

$K=K_{l} \cup K_{e}$. We define the projected relative capacity $\kappa_{b}^{i}$ as the available capacity of the vehicle before reaching the station $i$.

\subsection{Demand side}

\subsubsection{Demand characterisation}

The demand is the number of travellers between pairs of stations. A traveller $y$ is thus characterised by an exogenous fixed origin station $s_{i}(y) \in N$, and a fixed destination station $s_{j}(y) \in N$. In this paper, we consider that pairs, origin-destination pairs and legs refer to the same notion. We define $Q_{i j}(t)$ as the total number of travellers waiting for a vehicle at station $s_{i}$ in order to go to station $s_{j} . Q_{i j}(t)$ is a set of users ordered on the basis of their waiting time, so that users who have been waiting the 
longest can be prioritised. At the origin station, users are generated in simulation by their waiting time $w_{y}=0$.

The number of users in a station $s_{i}$ that are allowed - given relative capacity -to board a vehicle $k$ running to station $s_{j}$ is represented as $Y_{k i j}(t)$. It cannot exceed the number of empty seats in the vehicle $\kappa_{b}(t)$. When $Y_{k i j}(t)>0$, the corresponding travellers are reserved by the vehicle $k$, and leave $Q_{i j}(t) . Y_{k i}(t)=\sum_{j} Y_{k i j}(t)$ indicates the total users boarding at station $s_{i}, \underline{Y_{k i}}(t)$ the total users alighting at station $s_{i}$, and $\overline{Y_{k l}}(t-1)$ the total on-board users in $k$ going to a destination $s_{j} \neq s_{i}$. Finally, the total number of travellers on board the vehicle $k$ at the station is $s_{i}$, noted $\overline{Y_{k l}}(t)$, and verifies $\overline{Y_{k l}}(t)=\overline{Y_{k l}}(t-1)-\underline{Y_{k i}}(t)+Y_{k i}(t)$. Note specifically that $Y_{k i j}(t)$ become $\overline{Y_{k l j}}(t)$ when travellers board the vehicle $k$.

\subsubsection{Changes in user states}

When registered at the origin station, the corresponding user $y$ is created with a waiting time $w_{y}=0$. When a given vehicle $k$ decides to take $y$ on board, user $\mathrm{y}$ is considered to be reserved and leaves the booking procedure. His travel time is divided into an access time, waiting time and in-vehicle time $t_{y}^{k}$ The user exits the simulation after alighting. The diagram below follows user $y$ from arrival at station $i$ to destination $j$ and shows the user's progress in the successive sets.

\begin{tabular}{|c|c|c|c|c|c|}
\hline $\begin{array}{l}y \text { enter in the } \\
\text { system from } i \text { to } j \\
\qquad t=t_{0}\end{array}$ & \multicolumn{2}{|c|}{$t=t_{1}$} & \multicolumn{2}{|c|}{$t=t_{2}$} & $\begin{array}{l}y \text { exit the } \\
\text { system }\end{array}$ \\
\hline \multicolumn{2}{|c|}{ Waiting in station } & \multicolumn{2}{|c|}{ Reserved by $k$} & In vehicle & Arriving at \\
\hline Registration & \multicolumn{2}{|c|}{$k$ Itinerary choice } & \multicolumn{2}{|c|}{ Boarding in $k$} & Alighting from $k$ \\
\hline $\begin{array}{l}w_{y}=0 \\
Q_{i j}=Q_{i j}+y\end{array}$ & \multicolumn{2}{|c|}{$\begin{array}{l}\Delta t_{1}=t_{1}-t_{0} \\
w_{y}=\Delta t_{1} \\
t_{y}^{a}=0 \\
Q_{i j}=Q_{i j}-y \\
Y_{k i j}=Y_{k i j}+y\end{array}$} & \multicolumn{2}{|c|}{$\begin{array}{l}\Delta t_{2}=t_{2}-t_{1} \\
w_{y}=w_{y}+\Delta t_{2} \\
t_{y}^{a}=\Delta t_{2} \\
t_{y}^{v}=0 \\
\overline{Y_{k l j}}=\overline{Y_{k l}}+y \\
Y_{k i j}=Y_{k i j}-y\end{array}$} & $\begin{array}{l}\Delta t_{3}=t_{3}-t_{2} \\
t_{y}^{v a}=\Delta t_{3} \\
\overline{Y_{k \imath \jmath}}=\overline{Y_{k \imath \jmath}}-y\end{array}$ \\
\hline
\end{tabular}

Figure 1 Progression states of a user $y$ during the travel procedure 


\section{Centralised Service Layout}

In an independent vehicle service, Strategies are determined at vehicle level by the strategy calculation module detailed in Poulhès et al. (2018), although the strategy is outsourced to a dispatcher.

The diagram below shows the structure of the agent-based model of the proposed dispatching solution. In this model, users are only passive agents; they change their states as seen in the previous section and move between three locations: the origin station, the vehicle and the destination station. The smart agent is therefore the vehicle.

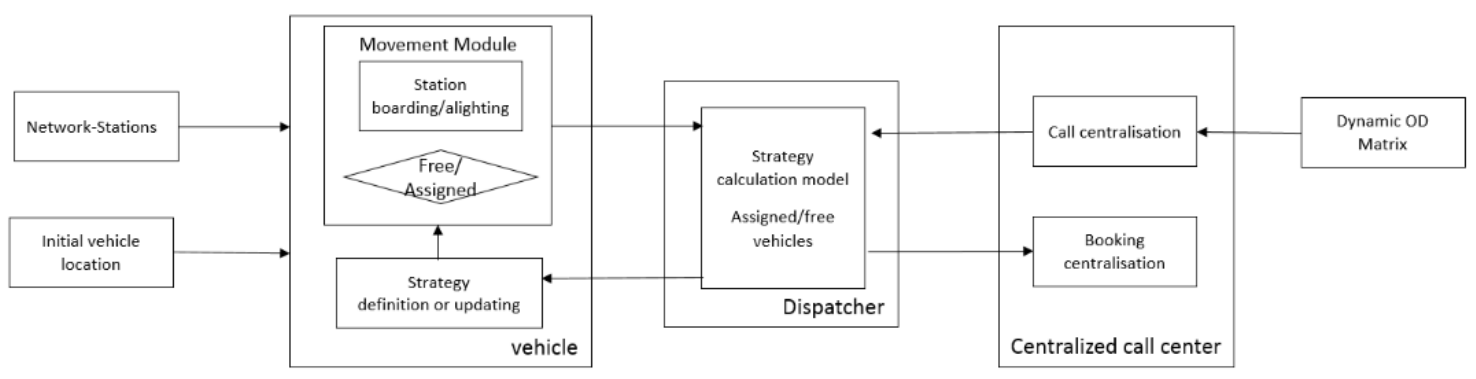

Figure 2 General scheme of the model with heterogeneous data and in case of dispatching

\subsection{Simulation period}

With the simulation period noted $\left[H_{0}, H_{0}+H\right]$, an initial loading period of simulation $\left[H_{-1}, H_{0}\right]$ provides realistic locations for the vehicles with destination and initial passenger load. A symmetrical post-period $\left[H_{0}+H, H_{1}\right]$ is introduced to assign the last user introduced in $\left[H_{0}, H_{0}+H\right]$ through to their destination. Indicators are calculated on users arrived at origin station in the simulation period. Similarly, indicators for vehicles come only from the simulation period.

\section{Centralised call centre}

The centralised call centre informs vehicles of a new call from a user, and updates the user bookings for each vehicle. The centre therefore manages the interface between users and vehicles. 


\subsection{Model scheme}

By contrast with a transit network, the itineraries of shared vehicles are unconstrained. In addition, they offer the advantage of easier coordination with demand in the vehicle network. At each iteration, in two steps, the strategy calculation dispatcher maximises a common strategy for assigned vehicles first and subsequently for free vehicles if residual demand exists.

Movement module: The movement module is active when the vehicle is assigned. It follows a calculated itinerary provided by a sequence of stations and links. Vehicle speed is considered constant, ignoring congestion effects. A vehicle's location is obtained from its itinerary and speed.

Strategy calculation module for dispatching service:

At each defined step, the dispatcher checks with the call centre to update demand. If there is non-assigned demand in the system, the dispatcher runs the strategy calculation module for the all assigned vehicles in order to optimise loading. This module tests the opportunity for all vehicles to board new users. It compares the results of all pertinent vehicle assignments for all vehicles simultaneously. Obviously, each vehicle only considers new demand when its projected residual capacity at the corresponding stations is positive. The best result is selected to assign demand. The optimal associated strategy is addressed to the vehicles concerned as new users and potential new stops. The dispatcher then assigns free vehicles to the closest and longest waiting demand.

Main assumptions:

- Assigned vehicles consider only demand generated and with a destination on their "pre-determined" itinerary. This means that the itinerary and destination are fixed only when the vehicle is free. Only 
new demand and intermediate stop stations can move dynamically at each step.

- The model considers detours only as extra intermediate stops in order to limit the spatial complexity.

- Fares are not included in the simulation. Fares mainly impact the level of demand and supply management, which are exogenous to our model. In the event of different levels of service and fares per vehicle types, demand segmentation will be introduced.

To sum up, the strategy, noted $S_{k}^{*}$, is defined as a combination of stations served $-N_{k}^{*} \in N-$ links travelled $-A_{k}^{*} \in A-$ and users reserved $-Y_{k}^{*} \in Q:$

$$
S_{k}^{*}=\left\{N_{k}^{*}, A_{k}^{*}, Y_{k}^{*}\right\}
$$

where users are represented by origin-destination pairs $\left\{y_{k i j}\right\} \in Y_{k i j},(i, j) \in$ $N^{* 2}$.

For instance, consider a simple network of three stations $(1,2,3)$. If the vehicle is located at station 1 with on-board demand going to station 3 , then there are different possible strategies depending on the demand to station 2 and $3:(1 \rightarrow 2),(2 \rightarrow 3),(1 \rightarrow$ $3)$ and $(1 \rightarrow 2 \rightarrow 3)$. The number of possible strategies is then dependent on the demand in stations and the relative capacity of the vehicle. If we describe the possible strategies as options, the vehicle has to test several options and choose the best one, which is its strategy. For instance, if we consider that the maximum demand allowed to board at each station is equal to $(5,3,0)$, then the optimal strategy is $S_{k}^{*}=$ $\left\{N_{k}^{*}, A_{k}^{*}, Y_{k}^{*}\right\}=\left\{\{1,2,3\},\left\{a_{12}, a_{23}\right\},\{1, \ldots, 8\}\right\}$. The problem of strategy calculation is therefore formulated as a problem of assessing options.

In the upcoming sections, we conduct an in-depth assessment of the options for a vehicle-centred approach (Section 4) and for a system-centred approach (Section 5). 


\section{Single vehicle strategy model}

In this section, we focus on the strategy calculated by a given vehicle independently of other vehicles. The main aim here is to maximise individual performance for trips completed by each vehicle. The vehicle's performance is evaluated through the notion of utility. The optimum strategy therefore corresponds to the option with maximum utility.

The section is organised as follows: first, we determine the set of options for assigned and free vehicles (4.1). Then, the utility is calculated for these options (4.2). Finally, the strategy for the vehicle is deduced (4.3).

\subsection{Option combination set per vehicle}

During operation time, demand is assigned dynamically. When the vehicle is assigned, the strategy depends on the destination of the on-board users. Therefore, the focus of the new strategy is to check new demand in stations on the vehicle's itinerary. In the case of free vehicles, all stations with residual demand are tested, and then demand along the path to the initial demand destination.

\subsubsection{Assigned vehicles}

For a vehicle $k \in K_{l}\left(\right.$ i.e. $\left.\kappa_{b}>0\right)$, let $N_{l_{k}}$ be the stations on its itinerary and specifically $N_{I_{k}}^{+}$the stations where demand is positive. This gives us $N_{l_{k}}^{+} \subset N_{l_{k}}$ and $N_{I_{k}}^{+}=\left\{i \in N_{I_{k}}\right.$, as $\left.Q_{i j}>0, j \in N_{I_{k}}\right\}$. As a result, the list of origin-destination pairs that will be considered by a vehicle is $p_{k}=(i, j) \in{N_{I_{k}}^{+}}^{+} \times N_{I_{k}}$ where $N_{I_{k}}^{+} \times N_{I_{k}}=$ $\left\{(i, j), i \in N_{I_{k}}^{+}, j>i, j \in N_{I_{k}}\right\}$. Finally, if the set of all tested $p_{k}$ is $\phi_{k}$ (i.e. $\phi_{k}=$ $\left.\left\{p_{k} \in N_{I_{k}}^{+} \times N_{I_{k}}\right\}\right)$, then an option is a subset of $\phi_{k}$, noted $o_{k}$. This gives us:

$$
o_{k}=\left\{\phi_{k}^{\prime} \text { as } \phi_{k}^{\prime} \subset \phi_{k}\right\}
$$

In addition, all the possible options for a vehicle $k \in K_{l}$ are listed in the set $O_{k}^{L}$. 
For instance, in a simple network of 3 nodes $(1,2,3)$, the legs are the set $\{(1,2),(1,3),(2,3)\}$. Supposing positive demand for all pairs, the set of all options $O_{k}^{L}$ could be written:

$\{((1,2)),((1,3)),((2,3)),((1,2),(1,3)),((1,2),(2,3)),((1,3),(2,3)),((1,2),(1,3),(2,3))\}$

The complexity of this vehicle-centred method of choice $O\left(C_{l_{k}}\right), k \in K_{l}$ is the number of combinations, $O\left(C_{l_{k}}\right)=\left(2^{\left|\phi_{k}\right|}-1\right)$. Then for all vehicles $K_{l}$, the vehicle with the highest complexity $O\left(C_{l_{k}}^{\max }\right)$ is multiplied by the number of assigned vehicles:

$$
O\left(C_{K_{l}}\right)=\left|K_{l}\right| . O\left(C_{l_{k}}^{\max }\right)
$$

\subsubsection{Free vehicle}

For free vehicles, which are not constrained by the destinations of on-board users, all stations with positive demand are explored as potential first stations to serve. They are noted $N^{0}$, where $N^{0} \subset N$. In concrete terms, the free vehicle takes the following steps in determining what option to adopt:

Step 1: All stations with positive demand are determined. They constitute the set $N^{0}$.

Step 2: For each station, we determine the set of user destinations. In particular, for a given station $m$, the set of desired destinations is $N^{0+}(m)$.

Step 3: After fixing the origin (Step1) and the destination (Step2), the intermediate stations on the itinerary are tested as in the case of assigned vehicles.

Specifically, if $N_{e_{k}}$ are the intermediate stations and $N_{e_{k}}^{+}$those with positive demand (i.e. $N_{e_{k}}^{+}=\left\{i \in N_{e_{k}}\right.$, as $\left.Q_{i j}>0, j \in N_{e_{k}}\right\}$ ), and $\phi_{k}^{e}$ is the set of all the origindestination pairs $p_{k}$, option $o_{k}$ is the subset defined as :

$$
o_{k}=\left\{m \in N^{0}, n \in N^{0+}(m), \phi_{k}^{\prime}(m, n) a s \phi_{k}^{\prime}(m, n) \subset \phi_{k}^{e}(m, n)\right\}
$$


Where, $\phi_{k}^{e}(m, n)$ is the selected legs on the path $\pi_{k m}+\pi_{m n}, \phi_{k}^{e}(m, n)=$ $\left\{(i, j) \in N_{e_{k}}^{+} \times N_{e_{k}}\right\}$

Work is now being done to restrict the space of possible options for exploration, which could be important in a large network. Carsharing systems (2012) or for vehicle routing problem (2012) research fields explore deeply this question.

All the possible options for the vehicle $k \in K_{e}$ are listed in the set $O_{k}^{E}$.

The complexity for one free vehicle is the complexity of $\phi_{k}^{e}(m, n)$, multiplied by the cardinal of $N^{0}$ and $N^{0+}(m), m \in N^{0}$

Thus for all free vehicles:

$$
O\left(C_{K_{e}}\right)=\left|K_{e}\right| \cdot\left|N^{0}\right| \cdot|N| . O\left(C_{e_{k}}^{\max }\right)
$$

\subsection{Option utility function for an assigned vehicle}

Vehicles choose passengers with the primary objective of maximising their total utility. We consider that the utility function increases with greater vehicle loading and significant user waiting time. This means that vehicles prefer users with a long waiting time or travel distance. These two main terms combine user and driver preferences. The utility function is attached to the vehicle but includes service and user considerations.

With our vehicle-centred approach, utility could be fixed by setting vehicle use parameters for each utility term. In the utility terms provided, for greater clarity the utility functions are presented without parameters. Price is not considered in the utility function. The path $\left(\pi_{i j}\right)$ between stations $i$ and $j$ on the graph $\mathrm{G}$ minimises travel time on the basis of Dijkstra's algorithm.

A singular strategy of stations served along a path is characterised by its utility in order to compare strategies. This utility function is divided into terms corresponding 
to pairs of stations. Each term is the sum of the total travel time and the total waiting time for all available users.

In addition, we detail the utility function for a given option $o_{k} \in O_{k}^{L} . o_{k}=$ $\left(p_{k}^{1}, \ldots, p_{k}^{n}\right),\left\{p_{k}^{1}, \ldots, p_{k}^{n}\right\} \in \phi_{k}$ for assigned vehicles or equally $o_{k} \in O_{k}^{E}$ for free vehicles. This subsection explains the utility calculation process with respect to demand per leg and the option for loaded flow between destinations. If the residual capacity is not sufficient to board all the demand at a station, the vehicle chooses the order of boarding priority for users on the basis of a utility term per user.

\subsubsection{Utility per leg}

Let us call a leg in the option $p_{k}=(i, j) \in o_{k}$. By definition, $\widetilde{Q}_{i j}>0$ is true for the demand from $i$ to $j$ when the strategy procedure is launched. The relative available capacity $\kappa_{b}^{i}$ before each station depends onthe number of users already reserved by the vehicle. $\kappa_{b}^{i}$ changes depending on $Y_{k i^{\prime} j},, \forall i^{\prime}<i, \forall j^{\prime}>i$. The demand also shares the total residual capacity for station $i$ with the other legs of the strategy beginning at $i$. The next part details the user selection method and the number of users actually loaded $Y_{k i j}$ for the option calculation.

Utility per leg consists of three terms:

- In the first, two perspectives are considered. Firstly, maximisation of occupancy rate and if necessary selection among users in the event of competition between destinations. If the number of passengers wishing to travel from $i$ to $j$ increases, the utility increases as well. Secondly, the preference for long paths without congestion $\left(\pi_{i j}\right.$ is the minimum path and $C\left(\pi_{i j}\right)$ the associated cost) 
- In the second, priority is given to the passengers who have been waiting the longest. It sums the waiting time of all selected passengers $Y_{k i j}$ in the stations going from $i$ to $j$ at the current time. The longer the waiting time, the greater the utility. We consider the penalty function $\alpha$ to calculate waiting time utility.

- However, the access time from the position of the vehicle $k$ to the station $i$ defined by the path $\pi_{k i}$, is a negative term for users.

Attributing weights $(\beta, \gamma, \theta)$ to each term on the basis of service management and strategies is a way to favour user or operating considerations. The utility $U_{k i j}$ for leg $(i, j)$ is:

$$
U_{k i j}=\beta \cdot\left|Y_{k i j}\right| . C\left(\pi_{i j}\right)+\gamma \cdot \sum_{y \in Y_{i j}} \alpha\left(w_{y}\right) \cdot w_{y}-\theta \cdot\left|Y_{k i j}\right| . C\left(\pi_{k i}\right)
$$

\subsubsection{Distribution of users per leg}

If the residual capacity of the vehicle is insufficient to board all interested demand, the vehicle needs to make a choice among users. The selection of users at a station among several destinations is a major strategy in all the service operator options. The operator can take a user-centred approach by favouring users who have been waiting the longest, or an operator-centred approach by simplifying loading operations and minimising the number of destinations among boarding users. We chose to adopt a uniform approach to the strategy, by selecting boarding users on the basis of their utility. For a user $y_{i j} \in \tilde{Q}_{i j}$ the utility for the service is:

$$
U_{y_{i j}}=C\left(\pi_{i j}\right)+\alpha\left(w_{y}\right) \cdot w_{y}
$$

Access time does not need to be included, as it is the same for all users at station $i$. 
The utility for all users on the legs starting at station $i$ and matching the option $o_{k}$ can be sorted into a list $L_{s_{k}}^{i}$. The users who will board the vehicle are at the top of this list. Their numbers are restricted by the residual capacity of the vehicle at station $i, \kappa_{b}^{i}$ : $\left|L_{s_{k}}^{i}\right| \leq \kappa_{b}^{i}$

An element $l$ of $L_{s_{k}}^{i}$, where the origin is $i$, the destination $j$, the user identifier $y_{i j}$, and the associated utility $U_{s_{k}}^{i}(l)$, is defined as:

$$
\begin{gathered}
L_{s_{k}}^{i}(l)=\left(i, j, y_{i j}, U_{y_{i j}}\right), y_{i j} \in \tilde{Q}_{i j}, \forall p_{k}=(i, j) \in o_{k} \mid U_{s_{k}}^{i}(l-1) \leq U_{s_{k}}^{i}(l) \\
\leq U_{s_{k}}^{i}(l+1)
\end{gathered}
$$

The users selected by origin-destination $(i, j)$ are the corresponding users $y_{i j}$ in the list $L_{S_{k}}^{i}$. This subset list is the set $Y_{i j}$ used in the utility calculation:

$$
Y_{k i j}=\left\{y_{i j}, U_{y_{i j}} \mid y_{i j} \in L_{s_{k}}^{i}\right\}
$$

\subsubsection{Utility function for the whole option}

We then sum the utility for all legs for a vehicle $k$ for a given service option. In order to favour a direct itinerary over a multi-stop route, we add a disutility term $D_{n}$ for time lost in new intermediate stops.

$D_{n}$ is calculated as the sum of access time, dwell-time and egress time. For station $n \in$ $N$, access/egress time is the additional time used in accessing and leaving the station, respectively termed $t_{n}^{a}$ and $t_{n}^{e}$. The dwell-time depends on the number of users boarding and alighting: $t_{n}^{d}=t_{u} \cdot\left(\sum_{j<n} Y_{k j n}+\sum_{j>n} Y_{k n j}\right)$, where a single unit $t_{u}$ is used for boarding or alighting time. Hence, $D_{n}=t_{n}^{a}+t_{n}^{d}+t_{n}^{e}$. Boarding and alighting time for stations already served are ignored. 
$Y_{n}$, the on-board flow during the dwelling procedure, is the impact on flow caused by detour, $Y_{n}=\sum_{i<n, j>n} Y_{k i j}$

$N_{k}^{-}$is the stops already programmed for an assigned vehicle, $N_{k}^{+}\left(o_{k}\right)$ the option stations obtained from the origin and destination stations for all legs of the option. The only new stations are $N_{k}^{+}\left(o_{k}\right)-N_{k}^{-}$.

Finally, where $(\beta, \gamma, \theta, \vartheta)$ are the service parameters, the utility $U_{o_{k}}$ for an option is:

$$
U_{o_{k}}=\sum_{p_{k} \in o_{k}} U_{p_{k}}(\beta, \gamma, \theta)-\vartheta \sum_{n \in N_{k}^{+}\left(o_{k}\right)} \mathbf{1}_{n \notin N_{k}^{-}} \cdot D_{n} . Y_{n}
$$

\subsection{Choice of Strategy}

As we saw previously, an option defines a list of stations served. The path is unchanged for assigned vehicles but defined by the first and last stop stations for free vehicles. The reserved users are, as we saw, chosen at the same time as the option.

\subsubsection{For assigned vehicle}

The selection strategy $S_{k}^{*}=\left\{N_{k}^{*}, A_{k}^{*}, Y_{k}^{*}\right\}$ corresponds to the option $o_{k}^{*} \in O_{k}^{L}$ with the maximum utility:

$$
U^{*}\left(o_{k}^{l *}\right)=\max _{o_{k} \in O_{k}^{L}} U_{o_{k}}
$$

\subsubsection{For free vehicle}

The selection strategy $S_{k}^{*}=\left\{N_{k}^{*}, A_{k}^{*}, Y_{k}^{*}\right\}$ corresponds to the option $o_{k} \in O_{k}^{E}$ with the maximum utility:

$$
U^{*}\left(o_{k}^{e *}\right)=\max _{o_{k} \in O_{k}^{E}} U_{o_{k}}
$$

In addition, $o_{k}^{e *}$ defines the first stop station $m^{*} \in S^{0}$ and the last stop station $n^{*} \in$ $S^{0+}(m)$. Thus the corresponding itinerary is the shortest path from the vehicle's 
position to the first stop station plus the path from the first stop station to the last one $A^{*} \sim \pi_{k m^{*}}+\pi_{m^{*} n^{*}}$

\section{Dispatcher solution}

We now go on to consider the case where vehicles cooperate through a dispatcher. We begin by presenting a general description of the service (5.1). Then, we describe the global option definition (5.2) and the utility function (5.3) for extreme sharing cases (i.e. where all options are independent or all options are common). Then, we investigate an optimized situation which combines shared and common options. In particular, we define the subset option definition (5.4.1) and the corresponding utility function (5.4.2). Finally, we present the optimal strategy defined for the particular case of free vehicles (5.5).

\subsection{Service description}

In a service with a central dispatcher, the vehicles share their planned itinerary and position. The operator's objective is therefore a global aggregate cost that reflects the social optimum for the service. This cost takes into account both the user's and the operator's interests. Drivers dependent on this service have no role in either demand management or strategy selection. They are purely restricted to the role of driving.

In a first step, we assign new users to already assigned vehicles. These kinds of vehicles have a fixed itinerary depending on the users on board. Potential new assigned users must have an origin and a destination on the vehicle's itinerary. Priority in assigning users to assigned vehicles is based on the objective of optimising the number of service vehicles and number of trips and reducing calculation time. Two assigned vehicles could have shared itineraries and thus be interested in the same potential users. In this case, we calculate the strategy of the two vehicles simultaneously. More 
generally, we construct groups of strategies as a combination of users served for a list of vehicles. The set of all combinations is evaluated and the solution adopted is the one with the least cost.

The second step is to assign the remaining demand to the free vehicles.

\subsection{Global option definition}

Each vehicle has its own list of options for legs travelled as defined in the previous section. The dispatcher's aim is to generate a combination of assignments between vehicles and users that optimises a shared utility function. We have already described the search procedure for a single vehicle. The easy way to test all the assignment possibilities between vehicles is to combine all the options for all the assigned vehicles. We now present solutions for reducing the option space.

Supposing that the assigned vehicles $K_{L}$ have a list of possible options named $O_{k}^{L}, k \in K_{L}$ precisely defined and calculated as in the previous section. The diagram in Figure 3 sums up all the options for each assigned vehicle. Some vehicles may have no available capacity or stations with positive demand on their path, with the result that the set $O_{k}^{L}$ is empty. For other assigned vehicles, a global option is a set of options with one option per vehicle. Two distinct global options must have at least one vehicle that has two different options in each.

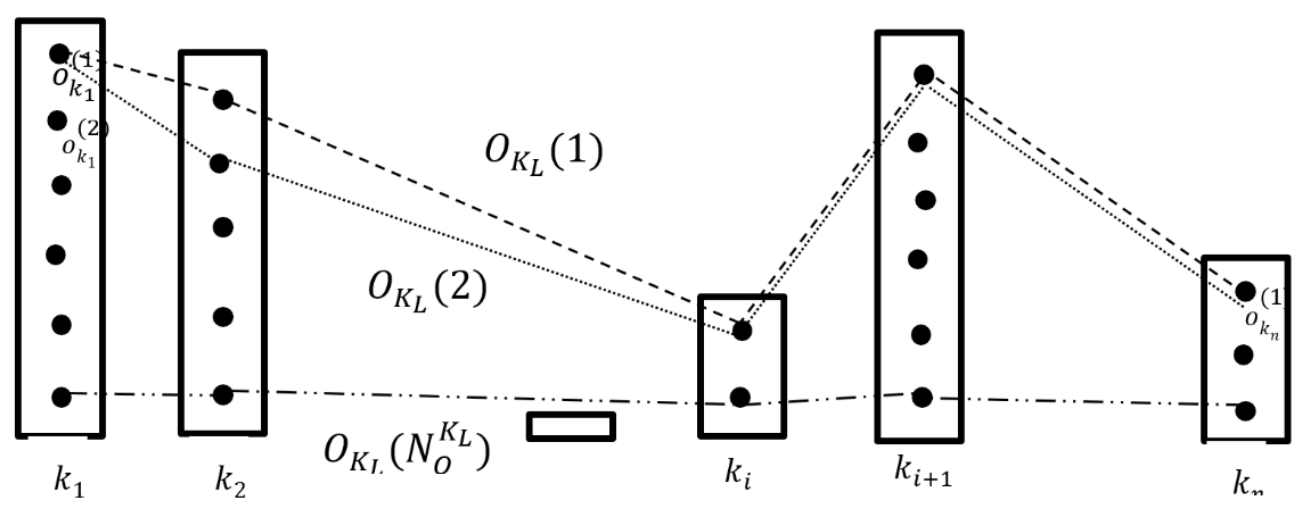


The total global option $N_{O}^{K_{L}}$ is the product of the number of options for each assigned vehicle, so $N_{O}^{K_{L}}=\prod_{k \in K_{L}}\left|O_{k}^{K_{L}}\right|$

If we note a global solution $O_{K_{L}}(r), r \in\left[0, N_{O}^{K_{L}}\right]$ :

$$
\begin{gathered}
O_{K_{L}}(r)=\left(o_{k_{1}}^{\left(i_{1}\right)} ; \ldots ; o_{k_{n}}^{\left(i_{n}\right)}\right), K_{L}=\left(k_{1} ; \ldots ; k_{n}\right),\left(i_{1}, \ldots, i_{n}\right) \\
\in\left(\left[1,\left|O_{k_{1}}^{K_{L}}\right|\right], \ldots,\left[1,\left|O_{k_{n}}^{K_{L}}\right|\right]\right)
\end{gathered}
$$

We group all the potential options $O_{K_{L}}$ for all assigned vehicles in a set:

$$
\Omega\left(\mathrm{K}_{\mathrm{L}}\right)=\bigcup_{r \in N_{O}^{L}} O_{K_{L}}(r)
$$

\subsection{Extreme sharing cases}

The utility for each vehicle leg is calculated as in the vehicle assignment solution. For a given option $o_{k} \in O_{k}^{K_{L}}, k \in K_{L}$, the associated utility is stated as $U_{o_{k}}$. The users assigned to a vehicle depend on the order of arrival at the station in the option scenario. The first vehicle $k(1)$ to arrive at a station $i$ can choose users $Y_{i j}^{k(1)}$ from all user demand $Q_{i j}, \forall j \in o_{k}$. The second vehicle can choose only users in $Q_{i j}-$ $Y_{i j}^{k(1)}, \forall j \in o_{k}$, and so on until the demand is zero on the leg.

A global option $O_{K_{L}}^{(r)}$ has by definition a total utility calculated as the sum of all vehicle utility:

$$
U_{O_{K_{L}}^{(r)}}=\sum_{o_{k} \in O_{K_{L}}(r)} U_{o_{k}}, k \in K_{l}
$$

\subsubsection{Naive formulation (100\% shared options)}

A dispatcher aims to find options for all vehicles by maximising the total utility of the available assigned vehicles $K_{l}$. The optimal global option $O_{K_{L}}^{*}(r)$ is given by: 


$$
U_{\Omega_{K_{L}}}^{*}=\max _{O_{K_{L}}^{(r)} \in \Omega_{S}}\left(U_{o_{K_{L}}^{(r)}, k \in K_{l}}\right)
$$

The optimal strategy for the vehicle $k \in K_{L}$ is the strategy corresponding to the option $O_{K_{L}}^{(r) *}, S_{k}^{*}=\left\{N_{k}^{*}, A_{k}^{*}, Y_{k}^{*}\right\}$

\subsubsection{Independent strategies (100\% independent options)}

The vehicle strategies are independent, in other words each origin-destination pair belongs to only one vehicle option set: ${ }^{1}$

$$
\forall\left(k, k^{\prime}\right) \in K_{L}^{2}, \forall o_{k} \in O_{k}^{K_{L}}, \forall o_{k^{\prime}} \in O_{k^{\prime}}^{K_{L}}, p_{k} \neq p_{k^{\prime}} \text { for } p_{k} \in o_{k}, p_{k^{\prime}} \in o_{k^{\prime}}
$$

In this case, each vehicle optimises its own strategy:

$$
U_{K_{L}}^{*}=\sum_{k \in K_{L}}\left(\max _{o_{k} \in O_{k}^{L}}\left(U_{o_{k}}\right)\right)
$$

For a vehicle $k \in K_{l}$ the assigned strategy $S_{k}^{*}=\left\{N_{k}^{*}, A_{k}^{*}, Y_{k}^{*}\right\}$ is the strategy with the highest utility among the options. This is the case we looked at in Section 4, where vehicles choose their strategies independently.

\subsection{Combined shared options}

The usual strategies contain vehicles with shared options and others with totally independent options.

If more than one vehicle are available for same users, a dispatcher optimises matching between users and vehicles. The total utility depends on the attribution choice. Users are assigned to vehicles in a way that maximises total utility. 
The cross strategies are evaluated together. The arrival order of vehicles in a station depends on the individual vehicle strategy. Two strategies can then pursue boarding solutions.

The naive strategy combination set is all cross combinations of the $K_{L}$ vehicles. $\left|S_{k}\right|=\left(2^{\left|\phi_{k}\right|}-1\right)^{\left|K_{L}\right|}$. We propose two main ways to reduce the size of the combination space.

\subsubsection{Subset definition}

The aim of this section is to subdivide the combination space into independent subgroups. Dispatching or choosing between vehicles are necessary only if several vehicles are available for the same demand, which means that sharing options, adopted where users match only one vehicle, are unnecessary.

From the vehicle point of view, on the one hand, the strategies of some vehicles may be totally independent, while on the other, some vehicles may have options that overlap with the options of other vehicles. In this case, the search space for the optimum strategy can be subdivided into several smaller subspaces. If a no-demand leg is shared between two vehicles, there is no need to combine all the strategies. The optimal strategy of the first vehicle and the optimal strategy of the second vehicle are the combined optimal strategy. Vehicles with shared legs are aggregated in the same group. Secondly, vehicles in the same group have their own strategies, totally independent of the other vehicles of the group. The strategies of all group vehicles on a shared leg are then combined into a single subgroup.

First, we create groups of dependent strategies. All the vehicles that have one leg in common belong to the same group. Consider the subgroups $K_{L}^{(l)} \subset K_{L}$ of vehicles to be $K_{L}=K_{L}^{(1)} \cup K_{L}^{(2)} \cup \ldots \cup K_{L}^{(l)}$. A subgroup $K_{L}^{(l)}$ is the set as: 


$$
\forall k \in K_{L}^{(l)},\left\{\begin{array}{c}
\forall k^{\prime} \in K_{L}^{(l)}, \exists\left(o_{k}, o_{k^{\prime}}\right) \in O_{k}^{K_{L}} \times O_{k^{\prime}}^{K_{L}} \mid \exists p_{k} \in o_{k}, \exists p_{k^{\prime}} \in o_{k^{\prime}}, p_{k}=p_{k^{\prime}} \\
\forall k^{\prime} \in K_{L} \backslash K_{L}^{(l)}, \forall\left(o_{k}, o_{k^{\prime}}\right) \in O_{k}^{K_{L}} \times O_{k^{\prime}}^{K_{L}} \mid \forall p_{k} \in o_{k}, \forall p_{k^{\prime}} \in o_{k^{\prime}}, p_{k} \neq p_{k^{\prime}}
\end{array}\right.
$$

For instance, consider the

Figure 4 . The vehicles $k_{1}$ and $k_{2}$ have the green leg in common. Then, they belong to the same group $K_{L}^{(2)}$. In addition, they are independent of $k_{3}$ and $k_{4}$.

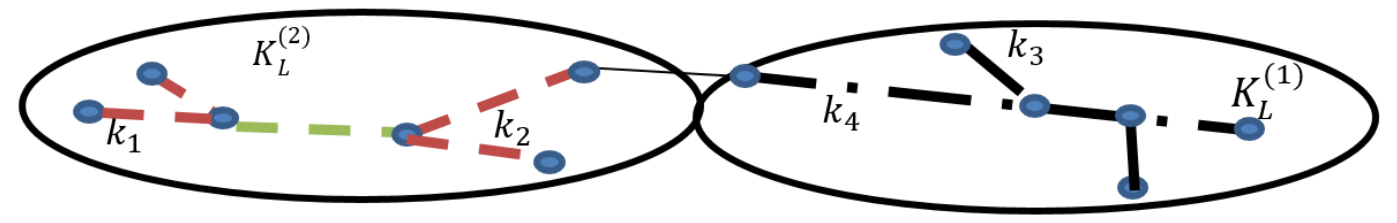

Figure 4 Illustration case for subset definition

We can then separate the optimal strategy into subgroups $K_{L}^{(l)}$ where $n$ is the number of subgroups:

$$
U_{\Omega_{K_{L}}}^{*}=\sum_{l \in[1, n]} U_{K_{L}^{(l)}}^{*}
$$

In each subgroup of $K_{L}: K_{L}^{(l)}$,two types of groups form the set $\Omega\left(K_{L}^{(l)}\right)$ :

(i) the group of common options. These, therefore, are the options with at least one leg in common. The group of cross options with one common leg through several vehicles $\widehat{\Omega}_{\mathrm{K}_{\mathrm{L}}^{(l)}} \subset \Omega$ is defined as

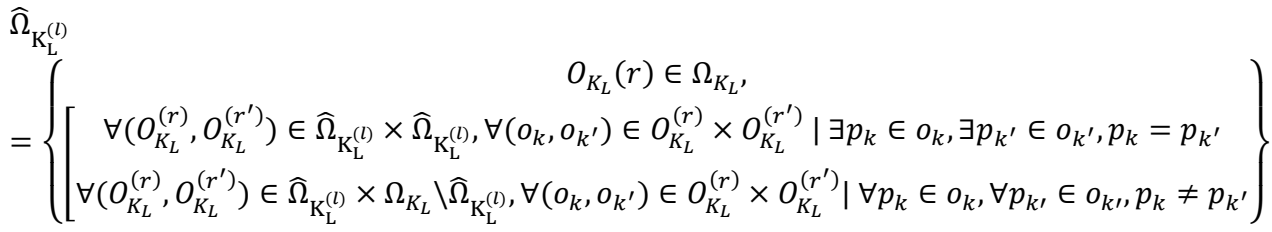

(ii) The complementary set represents the group of other single options per vehicle, $\bar{\Omega}_{\mathrm{K}_{\mathrm{L}}^{(1)}}=\Omega_{\mathrm{K}_{\mathrm{L}}} \backslash \cup \widehat{\Omega}_{\mathrm{K}_{\mathrm{L}}^{(1)}}$ defines as 


$$
\begin{aligned}
\overline{\boldsymbol{\Omega}}_{\mathbf{K}_{\mathrm{L}}^{(l)}}=\left\{\boldsymbol{o}_{\boldsymbol{K}_{L}}(\boldsymbol{r})\right. & \in \boldsymbol{\Omega}\left(\overline{\boldsymbol{K}_{L}}\right) \mid \forall\left(\boldsymbol{O}_{\boldsymbol{K}_{\mathbf{L}}}^{(\boldsymbol{r})}, \boldsymbol{o}_{\boldsymbol{K}_{L}}^{\left(r^{\prime}\right)}\right) \in \overline{\boldsymbol{\Omega}}_{\mathbf{K}_{\mathrm{L}}^{(l)}} \times \cup \widehat{\boldsymbol{\Omega}}_{\mathbf{K}_{\mathrm{L}}^{(l)}}, \forall\left(\boldsymbol{o}_{\boldsymbol{k}}, \boldsymbol{o}_{\boldsymbol{k}^{\prime}}\right) \\
& \left.\in \boldsymbol{O}_{\boldsymbol{K}_{\mathbf{L}}}^{(\boldsymbol{r})} \times \boldsymbol{O}_{\boldsymbol{K}_{\mathbf{L}}}^{\left(\boldsymbol{r}^{\prime}\right)}, \forall\left(\boldsymbol{p}_{\boldsymbol{k}}, \boldsymbol{p}_{\boldsymbol{k}^{\prime}}\right) \in \boldsymbol{o}_{\boldsymbol{k}} \times \boldsymbol{o}_{\boldsymbol{k}^{\prime}}, \boldsymbol{p}_{\boldsymbol{k}} \neq \boldsymbol{p}_{\boldsymbol{k}^{\prime}}\right\}
\end{aligned}
$$

The strategy with the maximal utility belongs to one of these two homogeneous groups of vehicles: $\bar{\Omega}_{\mathrm{K}_{\mathrm{L}}^{(1)}}$ and $\widehat{\Omega}_{\mathrm{K}_{\mathrm{L}}^{(l)}}$.

\subsubsection{Utility formulation of optimised strategies for a subgroup $K_{L}^{(l)}$}

Total utility can be defined as the maximum between two independent subgroups:

$$
U_{\mathrm{K}_{\mathrm{L}}^{(l)}, \Omega}^{*}=\max \left(U_{\widehat{\Omega}_{\mathrm{L}}^{(l)}}^{*}, U_{\bar{\Omega}_{\mathrm{K}}^{(l)}}^{*}\right)
$$

Within,

$$
\left\{\begin{array}{l}
U_{\widehat{\Omega}_{K_{L}^{(l)}}^{(l)}}^{*}=\max _{O_{K_{L}^{(r)} \in \widehat{\Omega}_{K_{L}^{(l)}}}\left(\sum_{o_{k} \in O_{K_{L}}^{(r)}} U_{o_{k}}\right)}\left(\max _{U_{K_{L}^{(l)}}^{*}}=\sum_{o_{K_{L}}^{(r)} \in \bar{\Omega}_{K_{L}^{(l)}}} U_{o_{k}} \in O_{K_{L}}^{(r)}\right)
\end{array}\right.
$$

In which the first utility $U_{\widehat{\Omega}}^{*}{ }_{K_{L}^{(l)}}$ is the maximum utility for shared options $O_{K_{L}}(r)$ in the $K_{L}^{(l)}$ vehicle group. And the second, $U_{\bar{\Omega}}^{*} K_{L}^{(l)}$ is the utility corresponding to the individual options of vehicles.

Vehicles in two groups belonging to $K_{L}$ are independent by construction. The global strategy is then the concatenation of all subgroup strategies.

For the dispatching method, the final maximal utility of assigned vehicles gives the strategy $S_{k}^{*}=\left\{N_{k}^{*}, A_{k}^{*}, Y_{k}^{*}\right\}, \forall k \in K_{L}$. 


\subsection{Free vehicle case}

Free vehicles are not restricted to a fixed itinerary. However, once a vehicle chooses a first target group of users on a leg, it is equivalent to an assigned vehicle. The first step is to match vehicles to remaining demand.

The demand remaining after assigned vehicles, called $\hat{Q}=\left\{\hat{Q}_{i j}>0,(i, j) \in\right.$ $\left.N^{2}\right\}$, should been assigned. A simple matching key is applied to limit tests between vehicles and demand. The number of free vehicles could be insufficient, so pairs $(i, j)$ are assigned to vehicle following the decrease in total waiting time: $W_{i j}=\sum_{y \in \hat{Q}_{i j}} w_{y}$.

Let $\hat{Q}_{i j}>0,(i, j) \in N^{2}$ be the number of users at the station $i$, and $K_{E}^{i j}$ the number of tested vehicles that verify the condition: all vehicles that have been parked less than $\tau_{i}$, a maximum fixed time for access to the station $i$, are tested. Otherwise, only the nearest vehicle to $i$ is tested:

$$
\begin{gathered}
K_{E}^{1}=\left\{k \in K_{E}, C\left(\pi_{k i}\right)<\tau_{i}\right\}, \text { if }\left|K_{E}^{1}\right|>0 \text { then } K_{E}^{i j}=K_{E}^{1} \text { else } K_{E}^{i j}=k \\
\in K_{E}, C\left(\pi_{k i}\right)=\min _{k^{\prime} \in K_{E}} C\left(\pi_{k^{\prime} i}\right)
\end{gathered}
$$

The optimum strategy for free vehicles can then be obtained on the basis of vehicles with initial demand. The case is the same as that of assigned vehicles with an initial itinerary and stations served.

\section{Study case presentation}

\subsection{Territory issues}

The model framework is applied to a French city located $17 \mathrm{~km}$ from the centre of Paris. It has a population of around 32,000 and provides some 22,000 jobs. The distribution of homes and jobs is heterogeneous. Palaiseau was chosen because of the French EVAPS project, headed by the VEDECOM Institute with a view to implementing a shared taxi service. Palaiseau is also becoming an area of interest 
because it is a part of a future French scientific cluster containing universities, graduate schools, research institutes and companies research labs. Today, Palaiseau is mainly connected to the rest of the Paris metropolitan area by the RER B train line. However, by 2030 the Greater Paris Express will provide public transport in the area through transit line 18.

\subsection{Network design}

A ridesharing service is provided in the area. It consists of a homogeneous fleet operating on a selected network that connects Massy-Palaiseau station at the eastern end to universities and research institutes located at the western end. The proposed network is designed to take into account the major spatial and demographic constraints of the area. It consists of 13 links and 11 stations (Figure 3 ). The arc cost is deduced directly from the length of the arc (e.g. in grey the Erreur ! Source du renvoi introuvable.) and the running speed of the vehicle. In the absence of congestion and other externalities, vehicle speed is assumed to be constant $(30 \mathrm{~km} / \mathrm{h})$.

\subsection{Demand generation}

The simulation is carried out for one morning peak hour and for home-to-work trips. Trips are estimated using a four-step model for the Paris area and then disaggregated by taxi stations by analysing the distribution of homes and jobs (TRB

accepted). The total number of trips is found to be equal to 600 passengers. Finally, trips are generated over time using a Poisson distribution per 1 minute time step for one hour of simulation. Vehicles are generated at station 1, which represents MassyPalaiseau station. 


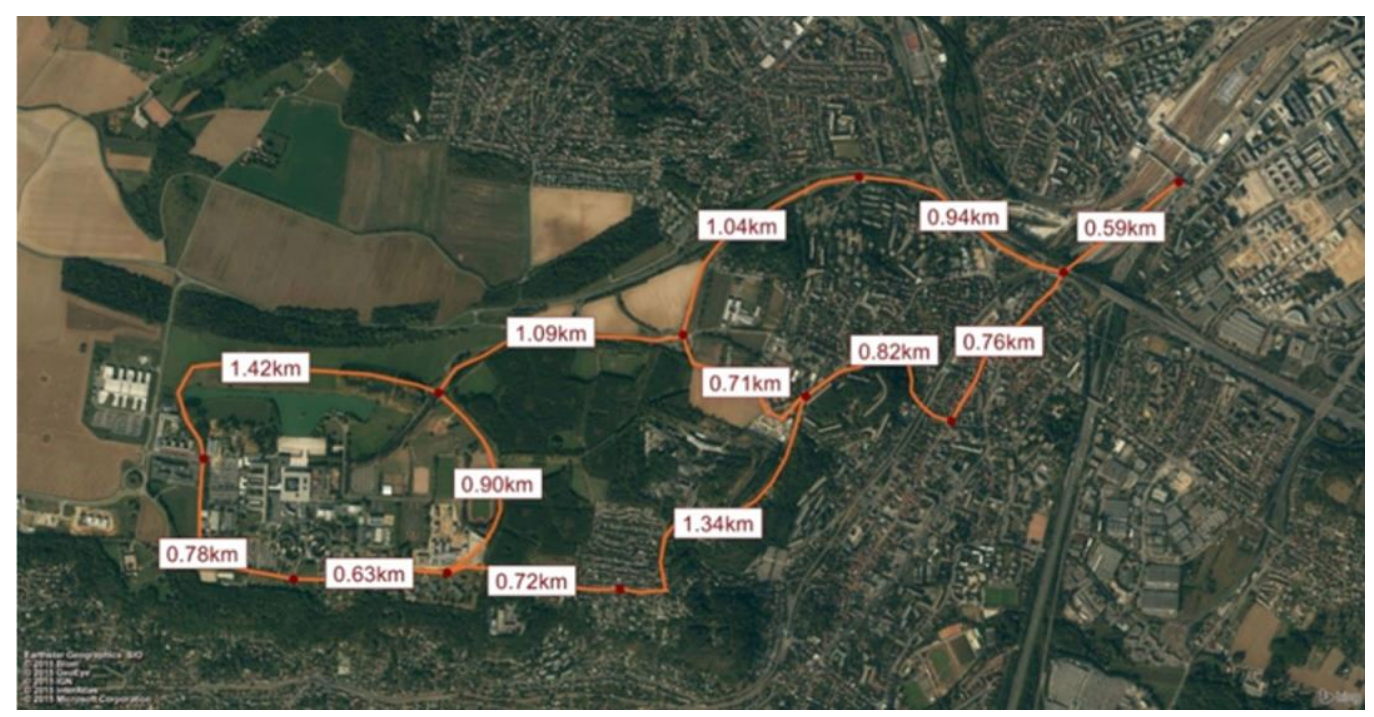

Figure 5 Drawing of the case study network

\subsection{Description of scenarios}

Our aim is to assess ridesharing service performances under the two assignment strategies, "single-vehicle strategy" and "dispatching strategy". For each strategy, we consider different fleet sizes and different levels of market penetration. The fleet size ranges between 10 and 40 vehicles. Market penetration is simulated by varying demand level: from $50 \%$ to $150 \%$ with a step of $25 \%$. These scenarios were combined with two capacity scenarios: (1) vehicles with 30 seats (e.g. minibuses, shuttles) and (2) vehicles with 5 seats (e.g. mid-sized cars). The weights of the utility function are fixed and are equal to 1 . This results in $2 *(4 * 2+5 * 2)=36$ scenarios.

\section{Simulation results}

\subsection{Operational performances}

Figure 6 depicts the empty time and flow-capacity ratio for the 36 scenarios defined. Empty time refers to the average ratio of time that vehicles are free during the study period. The flow-capacity ratio, on the other hand, relates the number of passengers using the service to the total capacity of the fleet. The assignment strategies 
are drawn in the same figures to allow intuitive comparison between their respective performances. Red crosses indicate extreme values.
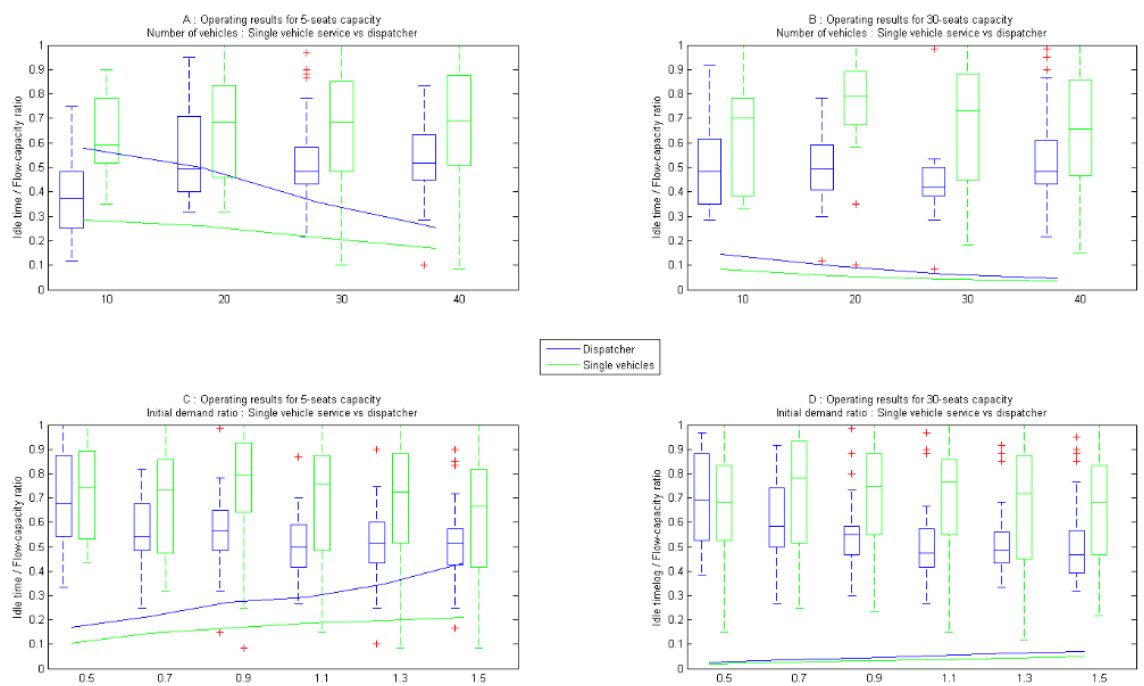

Figure 6 Operational performances with tests on operating condition and demand level

Figure 6.a shows that the dispatching strategy promises better results for all fleet sizes tested. In particular, the larger the fleet, the bigger the gap between the performances of the two strategies. For a fleet of 10 mid-sized vehicles, empty time for the single-vehicle strategy is almost double (60\%) that of the dispatching strategy (35\%). Increasing the number of vehicles results in higher empty times for both strategies, therefore leading to higher empty mileages, and hence higher operating costs. In addition, going from 10 to 40 vehicles, the values of the flow-capacity ratios for the two assignment strategies decrease and become increasingly close.

Figure 6.b confirms the results observed in Figure 6.a for 30-seat vehicles. It shows, however, that the gap between the two strategies in terms of empty time increases with fleet size, rising from $5 \%$ for 10 vehicles to $40 \%$ for 30 vehicles. Moreover, opting for high-capacity vehicles decreases the flow-capacity ratio significantly by comparison with mid-sized vehicle scenarios. 
Figure 6.c and 6.d present operational results for different levels of market penetration by fixing the fleet size at 40 vehicles. They show that increasing demand results in higher flow-capacity ratios and lower empty times. In particular, with lower demand levels, the two strategies produce almost the same operational performances. When demand grows, however, the dispatching strategy becomes more efficient. For instance, Figure 6.d shows that including the dispatcher reduces empty time by about 25\% when demand increases from between 300 to 900 passengers (i.e. for a multiplier of 0.5 and 1.5 respectively). The figures prove in addition that the greater the vehicle capacity, the lower the flow-capacity ratio and the bigger the gap between the two strategies.

\subsection{Quality of service performances}

Quality of service is measured by waiting time. Figure 7 depicts the maximum and average waiting times for different scenarios.

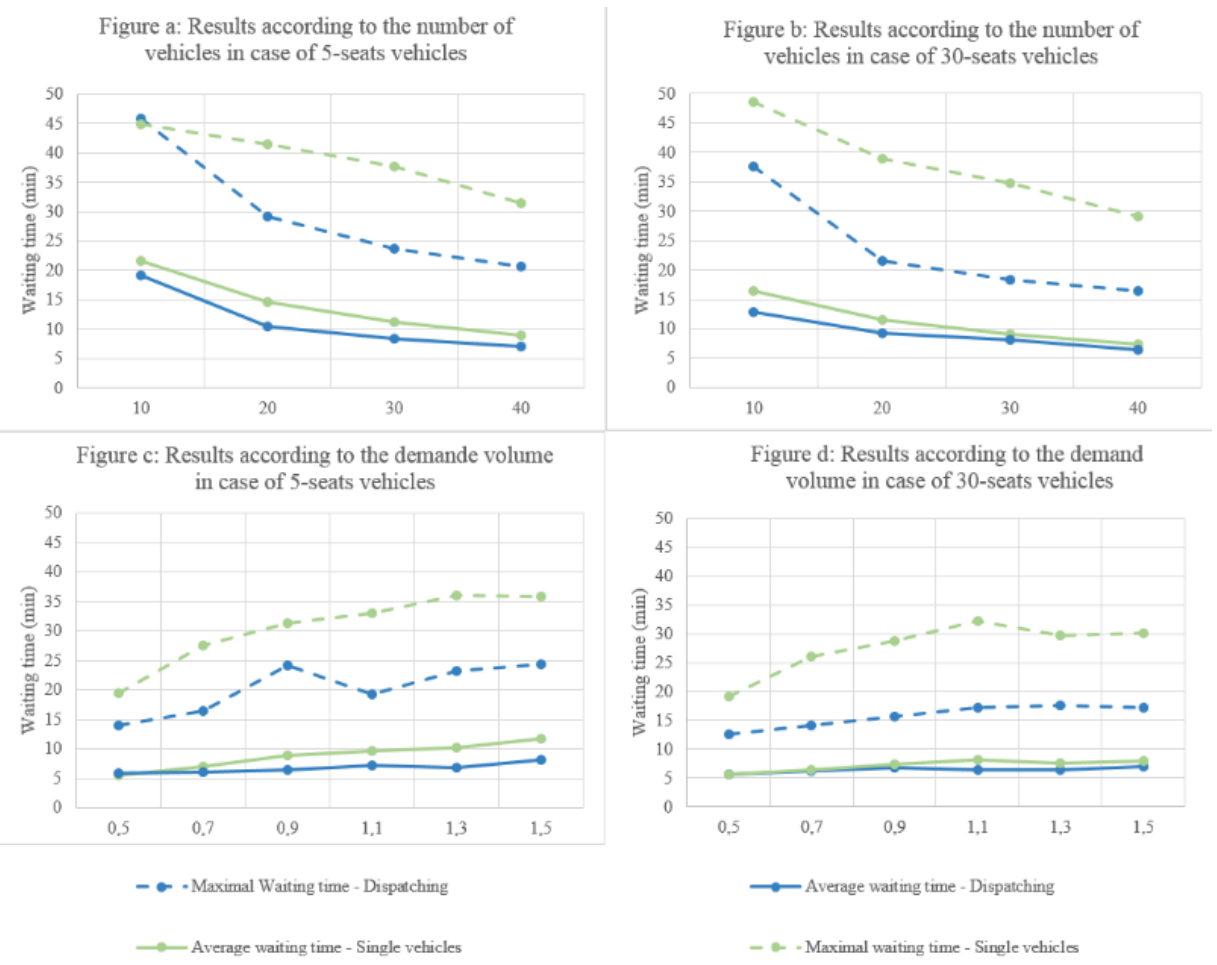

Figure 7 Quality of service with tests on operating condition and demand level 
Figure 7.a shows that increasing the fleet size improves the quality of service on both services. The comparison between the performance of dispatching and singlevehicle strategies confirms the findings of the operational analysis. However, the performance outcomes improve much faster in the case of dispatching. The coordination between vehicles appears more efficient when the number of vehicles increased. In particular, for a 40-vehicle scenario, the maximum waiting time with a single-vehicle strategy is 35 minutes, as compared with 20 minutes with a dispatcher. The average waiting time gain with dispatching varies by only some $10-20 \%$, while the maximum waiting time benefit can be close to $90 \%$. Increasing vehicle capacity (Figure 7.b) has little impact for the single-vehicle strategy, whereas the gain is around $20 \%$ for the dispatching strategy.

In term of demand sensitivity (Figure 7.c and Figure 7.d), having a fixed number of service vehicles reduces sensitivity to demand variation. The single-vehicle strategy is more sensitive, ranging with 5 -seat vehicles from $-40 \%$ for a 0.5 ratio to $+30 \%$ for a 1.5 demand ratio, while dispatching varies from $-20 \%$ to $+20 \%$. Obviously, 30 -seat vehicles provide a more robust service, and the performance is the same as 5-seat vehicles for a 0.5 demand ratio but significantly better for a 1.5 ratio, $-30 \%$ for average waiting time with dispatching or $-15 \%$ for the single-vehicle strategy. When demand is halved, some vehicles are almost unused, travelling less than $5 \mathrm{~km}$, while other vehicles travel an average of $25 \mathrm{~km}$.

Another major finding from Figure 7 is that the assignment strategy does not have a highly significant impact on average waiting time. The gap, widens, however, when demand increases with a fixed fleet size, and when the fleet size decreases for a given fixed demand volume. 


\subsection{Running time}

In this case study, our model is run by a Matlab program. For the single vehicle service, each hour of simulation takes 10 seconds, and 20 simulations were run for each scenario. The step time is 1 minute and the strategy calculation module is run every minute. Initial loading in terms of cars and users is included in the simulation. All vehicles start from the same station at the beginning of the simulation. The solution runs for between 30 seconds and 2 minutes, depending on the number of vehicles. The step time value has to be calibrated to two opposing phenomena: a shorter interval limits demand size and hence strategy space, meaning that vehicles are poorly optimised; on the other hand, a large interval increases user waiting time and restricts passenger information.

\section{Conclusion}

We described two theoretical models of dynamic ridesharing strategies in the context of a station network. These models are original in that they adopt a vehiclecentred approach that integrates user preferences into the utility function, with the result that optimisation can be comprehensively resolved through demand aggregated by origin-destination pair. In the first solution, the strategy decision is vehicle centred, while in the second, available vehicle options are shared via a central dispatcher. The model provides a primary framework for the testing of potential improvements. By choosing a station-based network, the demand is aggregated in specific points, hence avoiding an unjustified increase of the number of intermediate stops.

A real case study illustrates the simplification of a real fleet size adjustment with evaluation by performance indicators for both solutions. Dispatcher based vehicle assignment is clearly beneficial for users and operators but restrictive for drivers. In 
addition, the initial results show that a service with large-capacity vehicles is less efficient than a fleet of 5-seat vehicles, even in a network with high demand and limited station numbers. With a sufficient number of vehicles, an acceptable maximal waiting time value is achieved. If the only consideration in the utility function is waiting time, the maximum waiting time is 15 minutes.

There are, however, some limitations of this research work. One major limitation is the absence of detours in our first approach, mainly for reasons of optimisation of complexity. The possibility of detours introduces in addition uncertainties regarding both travel time and user waiting time. They also involve an increase of the number of intermediate stops, leading then to an exponential rise in the complexity of the calculation. Another major limitation is the estimation of the travel time while considering real operational condition such as the register time, the dispatcher response time and the waiting time. Another way in which the model could be developed would be to incorporate stochastic vehicle travel time to reflect traffic congestion. The model should also provide users with a predicted maximum waiting time. In addition, sensitivity analysis of the waiting time parameters is required. Last but not least, much of the current research uses big-city case studies, where the optimisation algorithms achieve an approximate match between users and vehicles, though falling short of the absolute optimum solution. On a small network, our aggregated demand approach enables us to achieve a precise optimum. In our future work, for large networks, compromises will need to be found between authorising detours and achieving optimum solutions (Agatz, et al., 2012). In addition, recoding our algorithm in a powerful programming language such as $\mathrm{C}++$ would make it possible to enlarge the network. 
In addition to recommendations mentioned above, future studies will need to investigate stations design issues. The location of stations would affect the performances of the service. Also, the capacity of stations would have impact on boarding/ alighting times, inducing perhaps significant extra-times. Therefore, deeper studies on operational design of stations according to the volume of the current demand and to the number of vehicles will improve the model and opens a new research field.

Finally, a standard DIAL a ride solution is competitive in a large network with low demand (Agatz, et al., 2012) while the present model operates in a structured network with high demand aggregated in a limited number of stations. This model of vehicles in a station network on shared roads can then be applied to various scenarios. Some public transport authorities such as Île de France Mobilité are thinking about an autonomous vehicle network connecting territories to metropolitan stations in place of a bus transit system. With calculated itineraries, such transport systems could run with autonomous vehicles replacing drivers. A socio-economic assessment will help to decide whether this kind of service would replace a bus transit system with complex origin-destination links. An in-depth economic analysis could compare the scenarios in terms of cost to users and operators and from a social perspective. Other possibilities that could be explored are user fare balancing, operating costs and benefits in term of air pollution, or benefits in accessibility. To sum up, this research could be considered as a starting point that compares technical performances of high capacity vehicles as buses and micro-transit services and mid-sized cars.

\section{References}

Agarwal, P. and K. Varadarajan. 2004. A near-linear constant-factor approximation for euclidean bipartite matching? Proceedings of the twentieth annual symposium on Computational geometry. 2004, pp. $247-252$.

Agatz, Niels, et al. 2012. Optimization for dynamic ride-sharing: a review. European Journal of Operational Research. December 2012, Vol. 223, 2, pp. 295-303. 
Agent Based Modelling for Simulating Taxi Services. Salanova, Josep Maria and Estrada, Miquel Angel. 2015. 2015, Procedia Computer Science, Vol. 52, pp. 902-907.

Alshamsi, Aamena, Sherief, Abdallah and Rahwan, Iyad. 2009. Multiagent Self-organization for a Taxi Dispatch System. Proceedings of 8th International Conference on Autonomous Agents and Mutiagent Systems. May 15, 2009.

Berbeglia, Gerardo, Cordeau, Jean-François and Laporte, Gilbert. 2010. Dynamic pickup and delivery problems. European Journal of Operational Research. April 2010, Vol. 202, pp. 8-15.

Boesch, Patrick, Ciari, Francesco and Axhausen, Kay. 2016. Required Autonomous Vehicle Fleet Sizes to Serve Different Levels of Demand. Proceeding of the 95th annual meeting of Transportation Research Board. 2016.

Burns, Lawrence, Jordan, William and Scarborough, Bonnie. 2013. Transforming Personal Mobility. New York, United States : The Earth Institute, Columbia University, 2013.

Cairns, Robert and Liston-Heyes, Catherine. 1996. Competition and regulation in the taxi industry. Journal of Public Economics. 1996, Vol. 59, pp. 1-15.

Cepolina, E. M. and Farina, A. 2012. Urban car sharing: an overview of relocation strategies. WIT Urban Transport XVIII. 2012.

Chen, Donna, Kockelman, Kara and Hanna, Josiah. 2016. Operations of a shared, autonomous, electric vehicle fleet: implications of vehicle \& charging infrastructure decisions. Proceeding of the 95th annual meeting of Transportation Research Board. 2016.

Cordeau, Jean-François and Laporte, Gilbert. 2007. The dial-a-ride problem: models and algorithms. Annals of Operations Research. September 2007, Vol. 153, pp. 29-46.

Effect of taxi information system on efficiency and quality of taxi services. Kim, H., Oh, J.D and Jayakrishnan. 2005. 2005, Transportation Research Record, Vol. 1903, pp. 96-104.

Fagnant, Daniel and Kockelman, Kara. 2014. The travel and environmental implications of shared autonomous vehicles, using agent-based model scenarios. Transportation Research Part C: Emerging Technologies. March 2014, Vol. 40, pp. 1-13.

Furuhata, Masabumi, et al. 2013. Ridesharing: The state-of-the-art and future directions. Transportation Research Part B: Methodological. November 2013, pp. 28-46.

Hackner, Jonas and Nyberg, Sten. 1995. Deregulating taxi services: a word of caution. Journal of Transport Economics and Policy. 1995, Vol. 29, 2.

Hörl, S., et al. 2017. Fleet control algorithms for automated mobility: A simulation assessment for Zurich. Proceedings of the annual meeting of transportation research board. 2017.

Hosni, Hadi, Naoum-Sawaya, Joe and Artail, Hassan. 2014. The shared-taxi problem: Formulation and solution methods. Transportation Research Part B: Methodological. December 2014, Vol. 70, pp. 303318.

Hyland, M. and Mahmassani, H.S. 2018. Dynamic autonomous vehicle fleet operations: Optimizationbased strategies to assign AVs to immediate traveler demand requests. Transportation Research Part C: Emerging Technologies. 2018, Vol. 92, pp. 278-297.

Lee, Der-Horng and Wu, Xian. 2013. Dispatching Strategies for the Taxi-Customer Searching Problem in the Booking Taxi Service. Proceedings of the annual meeting of Transportation Research Board. 2013.

Lioris, Eugénie. 2010. Evaluation et optimisation de syst emes de taxis collectifs en simulation. Paris : CERMICS, 2010.

Maciejewski, M., Bischoff, J., \& Nagel, K. 2016. An Assignment-Based Approach to Efficient RealTime City-Scale Taxi Dispatching. IEEE Intelligent Systems. 2016, Vol. 31, 1, pp. 68-77.

Maciejewski, Michal and Nagel, Kai. 2014. The Influence of Multi-agent Cooperation on the Efficiency of Taxi Dispatching. Conference: International Conference on Parallel Processing and Applied Mathematics. May 2014.

Nourinejad, Mehdi and Roorda, Matthew. 2014. Agent Based Model for Dynamic Ridesharing. Transportation Research Board 93rd Annual Meeting. Washington DC : Transportation Research Board, 2014. 01516547.

Poulhès A, Berrada J. 2018. User assignment in a smart vehicles' network: dynamic modelling as an agent-based model. Transportation Research Procedia. 2018.

Seow, Kiam Tian, Dang, Nam Hai and Lee, Der-Horng. 2010. A Collaborative Multiagent TaxiDispatch System. IEEE Transactions on Automation Science and Engineering. July 2010, Vol. 7, 3, pp. 607-616.

Taxi management and route control: a systems study and simulation experiment. Bailey, W.A. and Clark, T.D. 1992. 1992, Proceedings of the 24th conference on Winter simulation, pp. 1217-1222.

Yang, H., et al. 2010. Equilibria of bilateral taxi-customer searching and meeting on networks. Transport Research B. 2010, Vol. 44, pp. 1067-1083. 
Zeddini, B., et al. 2012. Managing Space-Time Networks For the Dynamic time-Constrained VRP. International Journal of Nonlinear Systems and Applications. 2012, pp. 88-97.

Zhang, Wenwen, et al. 2015b. Exploring the Impact of Shared Autonomous Vehicles on Urban Parking Demand: An Agent-Based Simulation Approach. Cambridge, Massachusetts, United States : s.n., $2015 \mathrm{~b}$.

- 2015a. The performance and benefits of a shared autonomous vehicles based dynamic ridesharing system: an agent. Washington DC, United States : s.n., $2015 \mathrm{a}$.

Zhu, Shirley and Kornhauser, Alain. 2017. The interplay between fleet size, level-of-service and empty vehicle repositioning strategies in large-scale, shared-ride autonomous taxi mobility-on-demand scenarios. Transportation Research Board 96th Annual Meeting. 2017. 